BULLETIN OF THE

AMERICAN MATHEMATICAL SOCIETY

Volume 79, Number 4, July 1973

\title{
A NOTE ON WITT RINGS
}

\author{
BY ANDREAS DRESS
}

Communicated by Hyman Bass, November 17, 1972

This note contains some applications of the theory of Mackey functors (cf. [3], [4] and [5]) to the study of Witt rings. A detailed version may be found in [3, Appendices A and B].

So let $R$ be a commutative ring with $1 \in R$ and $W(R)$ its Witt ring as defined in [7]. Any ring homomorphism $\rho: R \rightarrow R^{\prime}$ defines a ring homomorphism $\rho_{*}: W(R) \rightarrow W\left(R^{\prime}\right)$. Moreover if $R^{\prime}$ is separable over $R$ and finitely generated projective as an $R$-module (let $\rho$ be called admissible in this case), the trace map $R^{\prime} \rightarrow R$ defines a $W(R)$-linear map backwards: $\rho^{*}: W\left(R^{\prime}\right) \rightarrow W(R)$ (cf. [1] and [12], [13]). These observations lead easily to

Proposition 1. Let $\mathfrak{C}$ be the category with objects the commutative rings $R$ (with $1 \in R$ ) and with morphisms $\left[R^{\prime}, R\right]_{\mathbb{c}}=\left\{\rho: R \rightarrow R^{\prime} \mid \rho\right.$ admissible $\}$ (i.e. $(\mathbb{C}$ is dual to the category of commutative rings with admissible maps). Then the Witt ring construction defines a Mackey functor $W: \mathbb{C} \rightarrow \mathscr{A} b$, the category of abelian groups, together with a commutative, associative and unitary inner composition, given by the multiplication in the Witt ring.

CoRollary 1. Let $\rho: R \rightarrow R^{\prime}$ be admissible and $n \cdot 1_{W(R)} \in \rho^{*}\left(W\left(R^{\prime}\right)\right)$ for some $n \in N$. Then all the "Amitsur cohomology groups" $H^{i}\left(R^{\prime} / R, W\right)$ (i.e. the cohomology groups of the semisimplicial complex $0 \rightarrow W(R) \rightarrow W\left(R^{\prime}\right)$ $\rightrightarrows W\left(R^{\prime} \otimes_{R} R^{\prime}\right) \rightrightarrows W\left(R^{\prime} \otimes_{R} R^{\prime} \otimes_{R} R^{\prime}\right) \rightrightarrows(\cdots)$ are $n$-torsion groups, especially for $n=1$ they are all trivial.

ProOF. Apply the results of [5] to this special situation (they were found precisely to be applied right here!).

Examples of admissible maps $\rho: R \rightarrow R^{\prime}$ with $1_{W(R)} \in \rho^{*}\left(W\left(R^{\prime}\right)\right)$ have been given by Scharlau (cf. [12] and [3, Appendix A, Lemmas 2.3, 2.4, 2.5]).

As a rather special case we get this way:

Corollary 2 (CF. [11] AND [8]). Let $L / K$ be a finite Galois extension (of fields) of odd degree and with Galois group $G$. Then the natural action of $G$ on $W(L)$ has trivial (co)homology:

$$
\begin{aligned}
H^{0}(G, W(L)) & \cong H_{0}(G, W(L)) \cong W(K), \\
H^{i}(G, W(L))=H_{i}(G, W(L)) & =\hat{H}^{j}(G, W(L))=0 \quad(i \geqslant 1, j \in Z) .
\end{aligned}
$$

AMS (MOS) subject classifications (1970). Primary 16A63; Secondary 12G05.

Key words and phrases. Witt rings, quadratic forms, Mackey-functor, Burnside ring, induction of Witt rings, Galois cohomology of Witt rings, Amitsur cohomology of Witt rings. 
Let us concentrate further on finite separable field extensions:

For a field $K$ the category of finite, commutative separable $K$-algebras is (by Galois!) dual to the category $G^{\wedge}$ of finite $G$-sets, where $G$ is the (profinite) Galois group of $K$, thus $W$ defines a Mackey functor on $G^{\wedge}$ with a commutative, associative and unitary inner composition, i.e. a Green functor in the sense of $[3, \S 8]$. The theory of Burnside rings and Mackey functors can easily be extended from finite to profinite groups and, applied to $W$, then leads to

PROPOSITION 2. There exists a natural ring homomorphism of the Burnside ring $\Omega(G)$ onto $W(K)$ (defined by mapping any transitive $G$-set $G / U(U$ an open subgroup of $G$ ) in $\Omega(G)$ onto the element in $W(K)$, which is represented by the bilinear form $(L, t)$ with $L$ the fixed field of $U$, considered as an $K$-vector space, and $t: L \times L \rightarrow K:(a, b) \mapsto \operatorname{trace}_{L / K}(a \cdot b)$ the canonical bilinear map, associated with the separable extension $L / K)$, which in case char $K \neq 2$ is surjective, already restricted to $\Omega(\bar{G}) \subseteq \Omega(G)$ with $\bar{G}$ the Galois group of $K(\sqrt{ } a \mid a \in K)$ over $K$.

This at first explains the analogy between the prime ideal structure of Burnside rings (cf. [2] and [3, §5]) and Witt rings (cf. [9]). Furthermore Proposition 2, combined with the results of [9] and [3, §5], allows us to obtain easily the famous theorems of A. Pfister, concerning the ring theoretic structure of Witt rings, e.g. that all torsion in Witt rings is 2-torsion.

Proposition 3. Let $K$ be a field and $\rho_{i}: K \rightarrow L_{i}(i=1, \ldots, t)$ be finite separable field extensions of $K$, all contained in the finite Galois extension $E / K$.

Then:

(1) $(E: K)_{2} \cdot 1_{W(K)} \subseteq \bigcap_{i=1}^{t} K e\left(W(K) \stackrel{\rho_{i *}}{\rightarrow} W\left(L_{i}\right)\right)+\sum_{i=1}^{t} \operatorname{Im}\left(W\left(L_{i}\right) \stackrel{\rho_{i}^{*}}{\rightarrow} W(K)\right)$,

$$
(E: K)_{2} \cdot\left(\bigcap_{i=1}^{t} \operatorname{Ke}\left(\rho_{i^{*}}\right) \bigcap \sum_{i=1}^{t} \operatorname{Im}\left(\rho_{i}^{*}\right)\right)=0
$$

(with $(E: K)_{2}$ the maximal power of 2, dividing the degree $(E: K)$ ),

(3) $2^{n} \cdot 1_{W(K)} \in \sum_{1}^{n} \operatorname{Im}\left(\rho_{i}^{*}\right)$ for some power $2^{n}$ of $2 \Leftrightarrow$ any ordering of $K$ can be extended to at least one of $L_{i}(i=1, \ldots, t)$.

Corollary 3. If $L / K$ is a finite Galois extension with Galois group $G$, then all the groups $H^{i}(G, W(L)), H_{i}(G, W(L))$ and $\hat{H}^{j}(G, W(L))(i \geqq 1, j \in Z)$ are $(L: K)_{2}$-torsion groups.

Using J. A. Green's transfer theorem (cf. [6]) one gets furthermore: 
Corollary 4. Let $E / K$ be a finite Galois extension, $L / K$ a maximal subextension of odd degree and $F / K$ the minimal subextension in $L / K$ such that $L / F$ is normal (i.e. the fixed field of all $K$-automorphisms of $L$ ). Let $L_{1}, \ldots, L_{t}$ be a family of subextensions of $E / K$ with $L \subseteq L_{i}$, which contains the compositions $L \cdot L^{\tau}$ for any $\tau \in G=\operatorname{Gal}(E / K)$ with $L^{\tau} \neq L$.

Then the imbeddings $\rho_{i}: F \rightarrow L_{i}, \sigma_{i}: K \rightarrow L_{i}, \sigma: K \rightarrow F$ induce an isomorphism

$W(K) / \sum_{1}^{t} \operatorname{Im}\left(\sigma_{i}^{*}\right) \rightarrow W(F) / \sum_{1}^{t} \operatorname{Im}\left(\rho_{i}^{*}\right): a+\sum_{1}^{t} \operatorname{Im}\left(\sigma_{i}^{*}\right) \mapsto \sigma_{*}(a)+\sum_{1}^{t} \operatorname{Im}\left(\rho_{i}^{*}\right)$,

whose inverse is given by

$$
W(F) / \sum_{1}^{t} \operatorname{Im}\left(\rho_{i}^{*}\right) \rightarrow W(K) / \sum_{1}^{t} \operatorname{Im}\left(\sigma_{i}^{*}\right): b+\sum \cdots \mapsto \sigma^{*}(b)+\sum \cdots .
$$

Finally let us remark on a rather curious byproduct of these results: Let $G$ be the full Galois group of a formally real field and $H \leqq G$ an open subgroup of odd index. Then there exists an open subgroup $F \leqq H$ of index 2 , such that for any closed subgroup $U \leqq G$ of order 2 the number $\left|G / F^{U}\right|=|\{g F \mid U g F=g F\}|$ of $U$-invariant cosets of $F$ in $G$ equals $\left|G / H^{U}\right|+1$.

\section{REFERENCES}

1. F. R. De Mayer, The trace map and separable algebras, Osaka J. Math. 3 (1966), 7-11. MR 37 \#4122.

2. A. Dress, A characterization of solvable groups, Math. Z. 110 (1969), 213-217. MR 40 \# 1491.

3. - Notes on the theory of representations of finite groups. I: The Burnside ring of a finite group and some AGN-applications, Multicopied lecture notes, Bielefeld, 1971 .

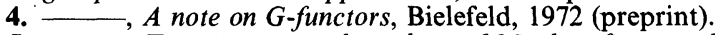

5. - Tate-Amitsur-cohomology of Mackey-functors, Bielefeld, 1972 (preprint).

6. T. A. Green, Axiomatic representation theory for finite groups, J. Pure Appl. Algebra 1 (1971), 41-77.

7. M. Knebusch, Grothendieck- und Wittringe von nichtausgearteten symmetrischen Bilinearformen, S.-B. Heidelberger Akad. Wiss. Math.-Natur. K. 1969/70, 93-157. MR 42 \#6001.

8. M. Knebusch und W. Scharlau, Ubber das Verhalten der Wittgruppe bei Körpererweiterungen, Math. Inst. der Universität Münster und Saarbrücken, 1970 (preprint).

9. J. Leicht und F. Lorenz, Die Primideale des Wittschen Ringes, Invent. Math. 10 (1970), 82-88. MR 42 \# 1851.

10. A. Pfister, Quadratische Formen in beliebigen Körpern, Invent. Math. 1 (1966), 116-132. MR 34 \# 169.

11. A. Rosenberg and R. Ware, The zerodimensional Galois cohomology of Wittrings, Invent. Math. 11 (1970), 65-72.

12. W. Scharlau, Zur Pfisterschen Theorie der quadratischen Formen, Invent. Math. 6 (1969), 327-328. MR 39 \#2793.

13. Induction theorems and the structure of the Wittgroup, Invent. Math. 11 (1970), 37-44.

Department of Mathematics, University of Bielefeld, Bielefeld, Federal RePUbliC OF GERMANY

Current address: 4814 Bielefeld Senne I, Brahmsstrasse 11, Federal Republic of Germany 